\title{
Humoral Response to Pfizer BNT162b2 Vaccine Booster in Maintenance Hemodialysis Patients
}

\author{
Moshe Shashara Naomi Nacasch $^{b, c} \quad$ Ayelet Grupper ${ }^{c, d}$ Sydney Benchetrit ${ }^{b, c}$ \\ Tamar Halperin ${ }^{e}$ Daniel Erez ${ }^{c, f}$ llan Rozenberg ${ }^{b, c}$ Pnina Shitrit ${ }^{c, g}$ \\ Yaron Sela $^{\mathrm{h}}$ Ori Wand ${ }^{\mathrm{c}, \mathrm{i}}$ Keren Cohen-Hagai ${ }^{\mathrm{b}, \mathrm{c}}$ \\ ${ }^{a}$ Department of Nephrology and Hypertension, Laniado Hospital, Netanya, Israel; bepartment of Nephrology \\ and Hypertension, Meir Medical Center, Kfar Saba, Israel; 'Sackler Faculty of Medicine, Tel Aviv University, Tel Aviv, \\ Israel; 'Department of Nephrology and Hypertension, Tel Aviv Sourasky Medical Center, Tel Aviv, Israel; 'Laboratory

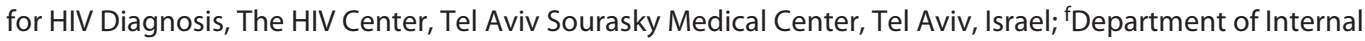 \\ Medicine D, Meir Medical Center, Kfar Saba, Israel; IInfection Control Unit, Meir Medical Center, Kfar Saba, Israel; \\ ${ }^{h}$ Interdisciplinary Center (IDC), Reichman University, Herzliya, Israel; 'Department of Pulmonology, Meir Medical \\ Center, Kfar Saba, Israel
}

\section{Keywords \\ Hemodialysis · COVID-19 virus · COVID-19 vaccine}

\begin{abstract}
Introduction: Coronavirus disease is associated with increased morbidity and mortality in maintenance hemodialysis (MHD) patients. Recent breakthrough infection in vaccinated people has led some authorities to recommend a booster dose for patients fully vaccinated 5-8 months ago. We aimed to assess the humoral response of MHD patients following a booster dose with the BNT162b2 vaccine. Methods: The study included 102 MHD patients vaccinated with 2 doses of the BNT162b2 (Pfizer-BioNTech) vaccine. A third dose (booster) was recommended to all MHD patients in our center and was given to those who opted to receive it, resulting in a booster group and a control group that did not receive the booster. Previous exposure was excluded by testing for the presence of the anti-nucleocapsid antibody (SARS-CoV-2) or positive PCR. We assessed the humoral response before and after the booster dose. Results: Of 66 patients in the booster group, 65 patients (98.5\%) developed a
\end{abstract}

positive antibody response, from $472.7 \pm 749.5$ to $16,336.8 \pm$ $15,397.3$, as compared to a sustained decrease in the control group $(695.7 \pm 642.7$ to $383.6 \pm 298.6), p<0.0001$. No significant adverse effects were reported. Prior antibody titers were positively correlated to IgG levels following the booster dose. There was a significant association between malnutrition-inflammation markers and the humoral response. Conclusions: Almost all MHD patients developed a substantial humoral response following the booster dose, which was significantly higher than levels reported for MHD patients following administration of 2 doses alone. Further studies and observations are needed to determine the exact timing and dosing schedule.

(C) 2022 The Author(s)

Published by S. Karger AG, Basel

\section{Introduction}

Coronavirus disease (COVID-19) caused by severe acute respiratory syndrome coronavirus 2 (SARS-CoV-2) is associated with increased morbidity and mortality in long-term hemodialysis patients [1]. The current CO-

C 2022 The Author(s).

Published by S. Karger AG, Basel

This is an Open Access article licensed under the Creative Commons Attribution-NonCommercial-4.0 International License (CC BY-NC) (http://www.karger.com/Services/OpenAccessLicense), applicable to the online version of the article only. Usage and distribution for commercial purposes requires written permission.
Correspondence to:

Keren Cohen-Hagai, keren.cohen@ clalit.org.il 
VID-19 pandemic is dominated worldwide by the Delta variant (B.1.617.2) [2]. The emergence of this variant has led to substantial breakthrough infections among vaccinated people. This has raised concern regarding vaccine efficacy versus new variants, as well as the possibility of waning immunity several months after being fully vaccinated. The recent emergence of the Omicron (B.1.1.529) variant has heightened this issue. A recent preprint from Israel suggests that the current COVID-19 wave, which occurred in a relatively vaccinated country, was caused by the combination of waning immunity from the vaccine, as well as emergence of a new variant (B.1.617.2) [3].

Observation of waning immunity, demonstrated by lower IgG S1 levels in vaccinated people, as well as the observation of a correlation between breakthrough infection and the time since being fully vaccinated $[4,5]$, has led medical authorities to recommend administration of a booster dose, first to patients over 65 years and other certain populations vaccinated more than 5 months ago [6]. Many studies have described the response of maintenance hemodialysis (MHD) patients to the various COVID-19 vaccines available and demonstrated a humoral response level which is less than that of healthy controls, but with a response rate of $80 \%-95 \%$ in this patient population $[7,8]$.

The current study was intended to establish the humoral response of the immune system, to the BNT162b2 (Pfizer-BioNTech) booster dose among MHD patients. Given the poor immune response in this cohort, we aimed to establish whether the booster dose can improve their humoral response in the face of both waning immunity and previous poor response to the 2-dose schedule. We determined the level of antibodies directed against the spike antigen (IgG S1), as well as the $\mathrm{N}$ antigen (previous infection) 6 months after receiving 2 doses and 2-3 weeks after the booster (third) dose of the Pfizer vaccine in patients on MHD and compared it to MHD patients who did not receive the booster dose.

\section{Material and Methods}

This observational study was conducted at the hemodialysis unit of Meir Medical Center, Kfar Saba, Israel, and included only patients with kidney disease treated with MHD, defined as at least 3 months of hemodialysis. Results are reported according to the STROBE statement guidelines.

The study was approved by the Ethics Committee and Institutional Review Board of Meir Medical Center (no. MMC-0029-21). Informed consent was obtained from all participants prior to study entry. The study was performed in accordance with the Declaration of Helsinki and Good Clinical Practice guidelines.

\section{Participants}

The study population included adults, aged $\geq 18$ years, on MHD who were able to provide written informed consent and were vaccinated with two doses of the BNT162b2 vaccine given 21 days apart. Patients were vaccinated in our dialysis unit or at their primary care clinic.

To determine the sample size, we used G-power software with the following assumptions: type 1 error of $5 \%$, minimum desired power of $90 \%$, and an expected moderate effect size for a difference in IgG S1 antigen between groups (Cohen's $d=0.7$ ). This calculation yielded a minimum sample size of 82 participants. A third (booster) vaccine dose was recommended for all MHD patients at the Meir Medical Center as part of the nationwide vaccination strategy in Israel, recommended by the Israeli Ministry of Health, and was administrated to all patients who opted to receive it. $\mathrm{Pa}$ tients who did not receive the booster dose served as the control group.

\section{SARS-CoV-2 Antibodies}

Levels of IgG S1 were measured twice using the AdviseDx SARS-CoV-2 IgG II Quant assay (Alinity, Abbott) on an Architect i200SR analyzer (Abbott). A cutoff value $\geq 50 \mathrm{AU} / \mathrm{mL}$ was considered a significant antibody response, as previously suggested [9].

Blood samples were drawn at the start of the hemodialysis sessions. Blood samples were taken 6 months after the first vaccine dose (June 2021, as shown in Fig. 1).

The second measurement was taken from all available study patients 8 months following the first dose of vaccine and 2-3 weeks after the booster dose was given. Anti-nucleocapsid antibody (anti-N Ab) (SARS-CoV-2) levels were measured in all study participants to exclude asymptomatic, covert infection. Anti-N Ab levels were measured using the Architect SARS-CoV-2 IgG nucleocapsid protein assay (Abbot, Abbot Park, North Chicago, IL, USA).

A diagnosis of SARS-CoV-2 infection was ascertained by a positive real-time reverse-transcriptase polymerase chain reaction result from a nasopharyngeal swab. A cycle threshold of 40 or less was considered positive. Patients were considered recovered if they had either positive PCR or positive anti-N Ab.

\section{Vaccine Schedule}

The first doses of BNT162b2 vaccine were administered on December 12, 2020, and continued through January 2021. Each patient received the second vaccine dose 21 days after the first dose.

Beginning on July 31,2021 , a third (booster) vaccine dose was recommended for all MHD patients at the Meir Medical Center and was given at our dialysis unit to all patients who opted to receive it. Patients were vaccinated in our dialysis unit or at their primary care clinic.

\section{Statistical Analysis}

Using G-power software, we conducted a power analysis that resulted in a minimum sample size of 82 patients. Descriptive statistics are presented as means, with standard deviation and range, median, or percentages. Continuous variables were examined for normality (Shapiro-Wilk test) and data were analyzed, accordingly. The $t$ test or one-way ANOVA was used for normally distributed variables and the Mann-Whitney or Kruskal-Wallis test for nonparametric variables. Variables between two study groups were compared using the $t$ test, Mann-Whitney test, Fisher's exact test, or $\chi^{2}$ test, according to the scale of measured variables. $p$ values 


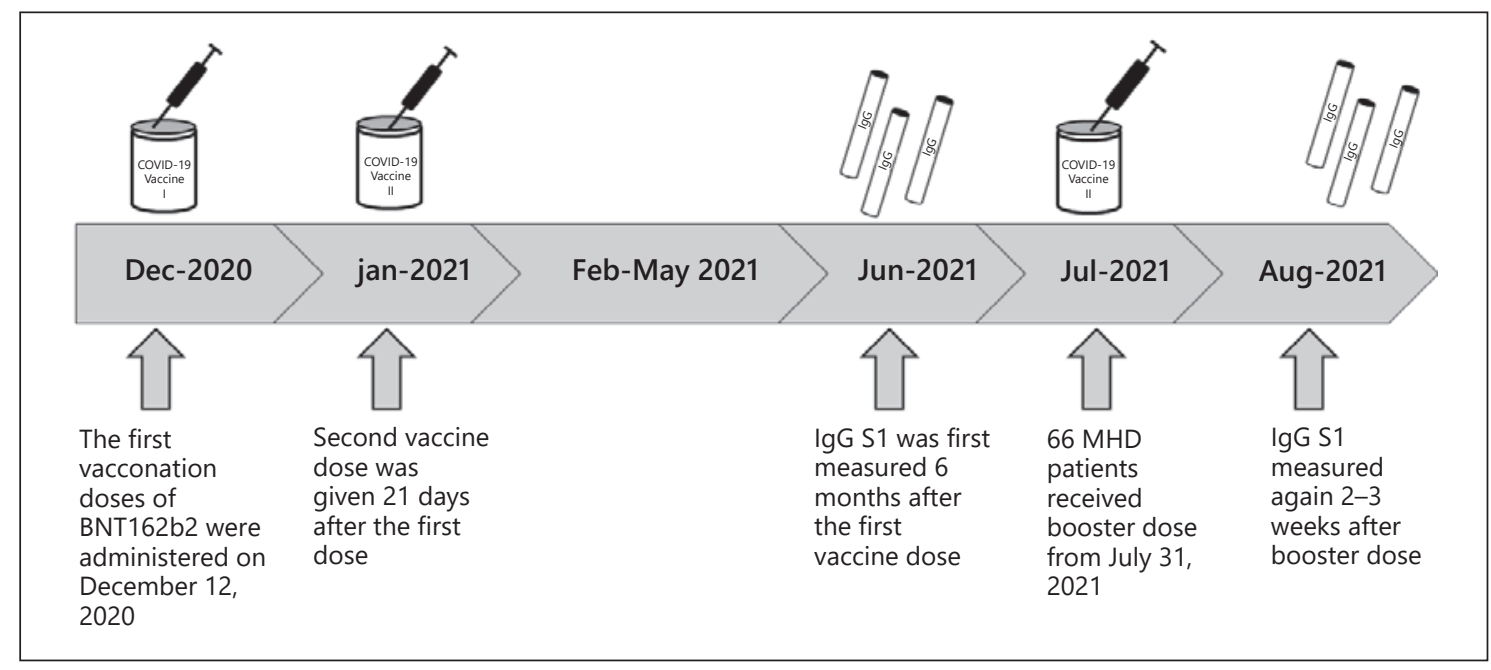

Fig. 1. Timeline of BNT162b2 vaccine schedule and measurements of IgG anti-Spike SARS-CoV-2 IgG anti-Spike SARS-CoV-2, IgG S1.

$<0.05$ were considered statistically significant. A box-plot describing median, 25th and 75th percentile, minimum, and maximum levels of the difference in IgG S1 titers between study groups was constructed using SPSS. To assess predictors of IgG S1 improvement, linear regressions were employed and a multivariate model adjusting for all possible confounders and variables for predicting the delta in humoral response was created. Data were analyzed using SPSS, Version 27 (IBM Corp., Armonk, NY, USA).

\section{Results}

The study included $102 \mathrm{MHD}$ patients who had received 2 doses of the BNT162b2 (Pfizer-BioNTech) vaccine. Ten patients tested positive for COVID-19 by PCR or anti-N Ab and were excluded from the study. Four additional patients were lost to follow up. A total of 88 patients who were not exposed to COVID-19 and who were available for two IgG S1 measurements were included in this analysis. They were referred to as the control group if they did not receive the booster dose $(n=22)$ and as the booster group $(n=66)$ if they received a booster dose at least 7 days prior to the IgG S1 measurement. No significant adverse effects were reported after the booster dose injection.

The first IgG S1 titers were measured $179.2 \pm 18$ days after the first vaccine dose and $158 \pm 17.4$ days after the second vaccine dose (BNT162b2, Pfizer-BioNTech). The mean IgG S1 in the entire cohort at the first time point was 529.1 $\pm 726.9 \mathrm{AU} / \mathrm{mL}$ (median 204, range 0-3,496.6 $\mathrm{AU} / \mathrm{mL})$.

Response to BNT162b2 Vaccine Booster in Hemodialysis Patients
Initial baseline IgG S1 titers were comparable between the control and booster groups $(695.7 \pm 642.7$ vs. $472.6 \pm$ $749.5, p=0.215)$. Patients in the booster group were older with higher prevalence of hypertension (Table 1).

Among the study cohort, 16 (18.1\%) MHD patients had IgG S1 titers below $50 \mathrm{AU} / \mathrm{mL} 6$ months after 2 doses of BNT162b2 vaccine. IgG S1 titers were measured a second time $17.2 \pm 3.6$ days after the booster dose.

\section{Substantial Humoral Response following the Booster Dose}

MHD patients in the booster group had significantly increased IgG S1 titers (from a median of $141.3 \mathrm{AU} / \mathrm{mL}$ to a median of $10,374.1 \mathrm{AU} / \mathrm{mL}$; mean elevation of $15,871.3 \mathrm{AU} / \mathrm{mL}$ with $95 \%$ confidence interval of 12,229 $19,777.9 ; p<0.0001)$. IgG S1 titers were significantly decreased in the control group (median decrease of 147.3 $\mathrm{AU} / \mathrm{mL}$, range $-1,421.2$ to $0 \mathrm{AU} / \mathrm{mL}, p<0.001)$. Individual variations in IgG S1 titers of control and booster groups are shown in Figure 2 and mean titers and variations between the groups are shown in Table 2.

\section{Factors Associated with the Humoral Response in the}

Booster Group

IgG S1 level at the second time point significantly correlated with first IgG S1 measurement $(p=0.025)$. Humoral response to the booster was positively correlated with albumin $(r=0.37, p=0.002)$ and inversely correlated with C-reactive protein (CRP) $(r=-0.348, p=$ 0.008). 
Table 1. Clinical characteristics of control and booster groups

\begin{tabular}{|c|c|c|c|}
\hline Characteristic & $\begin{array}{l}\text { Control group } \\
(N=22)\end{array}$ & $\begin{array}{l}\text { Booster group } \\
(N=66)\end{array}$ & $p$ value \\
\hline Age, years & $57.9 \pm 16.9$ & $75 \pm 10.2$ & $<0.0001$ \\
\hline Male sex & $18(81.8)$ & $39(59.1)$ & 0.05 \\
\hline Dialysis vintage, months & $29.8 \pm 31.2$ & $30.4 \pm 23$ & 0.9 \\
\hline 6-month lgG S1 & $695.7 \pm 642.7$ & $472.6 \pm 749.5$ & 0.2 \\
\hline Negative IgG S1 titer & $3(13.6)$ & $13(19.6)$ & 0.5 \\
\hline \multicolumn{4}{|l|}{ Comorbidities } \\
\hline Diabetes mellitus & $13(59.1)$ & $43(65.2)$ & 0.6 \\
\hline Hypertension & $13(59.1)$ & $57(86.4)$ & 0.006 \\
\hline Ischemic heart disease & $4(18.2)$ & $26(39.4)$ & 0.07 \\
\hline Heart failure & $3(13.6)$ & $23(34.8)$ & 0.06 \\
\hline Peripheral vascular disease & $0(0)$ & $9(13.6)$ & 0.07 \\
\hline Chronic lung disease & $2(9.1)$ & $10(15.2)$ & 0.5 \\
\hline Active malignancy & $1(4.5)$ & $2(3)$ & 0.7 \\
\hline Chronic immunosuppressive therapy & $3(13.6)$ & $3(4.6)$ & 0.1 \\
\hline \multicolumn{4}{|l|}{ Baseline laboratory data } \\
\hline WBC, $\mathrm{K} / \mu \mathrm{L}$ & $6.6 \pm 2.2$ & $6.3 \pm 1.7$ & 0.5 \\
\hline Hemoglobin, $\mathrm{g} / \mathrm{dL}$ & $10.7 \pm 1.4$ & $10.6 \pm 1.1$ & 0.6 \\
\hline 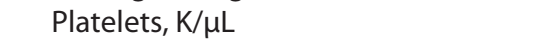 & $192 \pm 69$ & $186 \pm 68$ & 0.8 \\
\hline Urea, mg/dL & $121 \pm 35$ & $122 \pm 35$ & 0.9 \\
\hline Serum creatinine, $\mathrm{mg} / \mathrm{dL}$ & $8.3 \pm 2.9$ & $6.7 \pm 1.9$ & 0.005 \\
\hline Total calcium, mg/dL & $8.5 \pm 0.8$ & $8.5 \pm 0.7$ & 0.9 \\
\hline Phosphorus, mg/dL & $5.3 \pm 1.8$ & $5.3 \pm 1.3$ & 0.9 \\
\hline Serum albumin, g/dL & $3.6 \pm 0.6$ & $3.6 \pm 0.3$ & 0.8 \\
\hline $\mathrm{CRP}, \mathrm{mg} / \mathrm{dL}$ & $1.8 \pm 3.3$ & $1.6 \pm 2$ & 0.7 \\
\hline Total cholesterol, mg/dL & $147 \pm 40$ & $138 \pm 38$ & 0.3 \\
\hline $\mathrm{Kt} / \mathrm{V}$ & $1.23 \pm 0.22$ & $1.3 \pm 0.22$ & 0.2 \\
\hline URR & $65.9 \pm 6.3$ & $67 \pm 7$ & 0.5 \\
\hline
\end{tabular}

Baseline laboratory values were taken at hemodialysis initiation 6 months following first vaccine dose. Negative IgG S1 titer considered as IgG S1 lower than 50 AU/mL 6 months after 2 doses of BNT162b2 vaccine. Values are presented as absolute $n(\%)$ or as mean \pm SD. WBC, white blood count; URR, urea reduction ratio.

Table 2. Mean IgG S1 titers in study groups

\begin{tabular}{lllr}
\hline Measurement & Control group $(n=22)$ & Booster group $(n=66)$ & $p$ value \\
\hline 6 months IgG S1 & $695.7 \pm 642.7$ & $472.6 \pm 749.5$ & 0.2 \\
8 months IgG S1 & $383.6 \pm 298.6$ & $16,336.8 \pm 15,397.3$ & $<0.0001$ \\
Mean difference between measurements & $-312.1 \pm 373.3$ & $15,871.3 \pm 15,152.9$ & $<0.0001$ \\
\hline
\end{tabular}

Values are presented as mean $\pm \mathrm{SD}$ in $\mathrm{AU} / \mathrm{mL}$ units.

Median albumin level in our cohort was $3.7 \mathrm{~g} / \mathrm{dL}$ (range 2.2-4.5 g/dL). Despite comparable dry weights, dialysis adequacy parameters and baseline IgG titers among patients with hypoalbuminemia (defined as serum albumin $<3.5 \mathrm{~g} / \mathrm{dL}$ ) had inferior response to the booster dose (mean elevation of 6,300.1 $\pm 6,601.7 \mathrm{AU} /$ $\mathrm{mL}$ vs. $18,636.7 \pm 15,900.8 \mathrm{AU} / \mathrm{mL}, p=0.011$; IgG S1 titer after booster 6,771.3 $\pm 6,814.2$ vs. $19,126 \pm 16,165.7 \mathrm{AU} /$ $\mathrm{mL}, p=0.012$ ).

Median CRP in our cohort was 0.9 (range 0-9.3 mg/ dL, upper limit 1.2). Despite comparable age and baseline IgG S1 titers before the booster, patients with high CRP had significantly inferior response to the booster (mean elevation of $10,881 \pm 14,126.5$ vs. $20,067.5 \pm 15,398.8 \mathrm{AU} /$ 


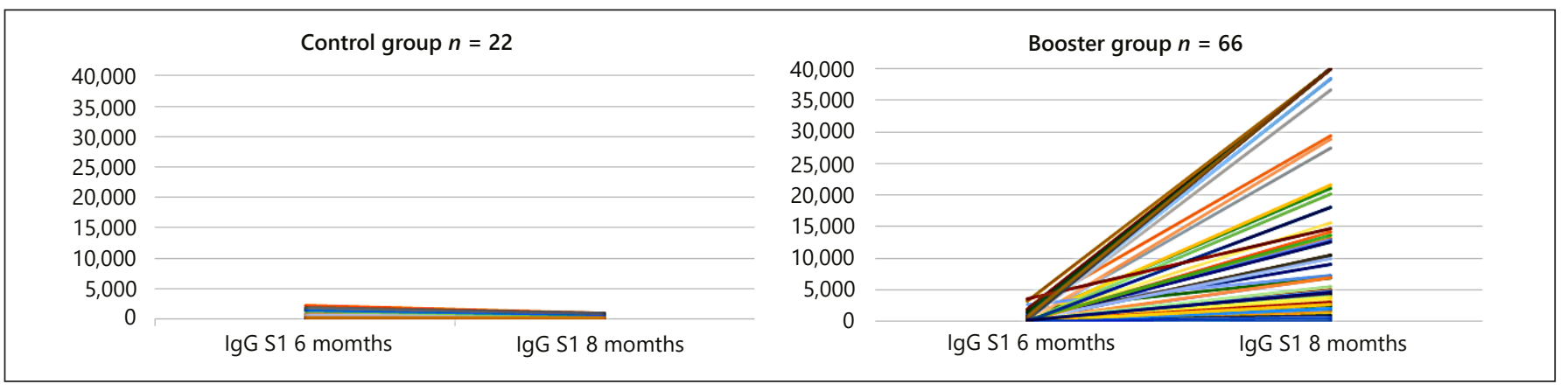

Fig. 2. COVID-19 IgG S1 titers in control and booster groups. 65/66 MHD in the study group had a significant increase in their IgG S1 titer: from $621 \pm 1,472.8$ to $16,451 \pm 15,548.5 \mathrm{AU} / \mathrm{mL}, p<0.0001$; mean increase of 15,871.3, with 95\% CI of 12,146.2-19,596.3 AU/mL; range 0-39,900; median 9,966). One patient had no change in titers. Antibody levels in the control groups declined a mean decrease $312.1 \pm 373.3 \mathrm{AU} / \mathrm{mL}$.

$\mathrm{mL}, p=0.027$; IgG titer after booster $11,307.3 \pm 14,324.1$ vs. $20,567.3 \pm 15,671.4 \mathrm{AU} / \mathrm{mL}, p=0.028)$. There was no association between sex, comorbidities (diabetes, ischemic heart disease, heart failure, chronic lung disease, or vascular disease), KT/V, dry weight, and antibody response.

\section{Age}

The median age of our cohort was 72.7 years (range 24.8-94.3) years. Age was inversely correlated with IgG titers at the first time point, in the booster group ( $r=$ $-0.672, p<0.0001)$ and in the control group $(r=-0.642$, $p=0.001)$.

Humoral response among elderly MHD patients after the booster was significantly increased, compared to decreasing titers in the control group, despite comparable titers at baseline. IgG S1 titers increased with the booster in all age groups with no significant difference between them. Among MHD patients older than 60 years in the study group, IgG S1 titer increased from $426.8 \pm 686.2$ $\mathrm{AU} / \mathrm{mL}$ to $16,700.2 \pm 15,433.7 \mathrm{AU} / \mathrm{mL}$ compared to the age-matched control group titer that decreased from $270.6 \pm 507.5 \mathrm{AU} / \mathrm{mL}$ to $171 \pm 263.6 \mathrm{AU} / \mathrm{mL}(p=0.006)$.

\section{Immunosuppressive Treatment}

Six patients were treated with immunosuppression therapy: one with steroids due to systemic lupus erythematosus; two were treated with a combination of bortezomib, cyclophosphamide, and dexamethasone due to multiple myeloma; one with rituximab due to idiopathic membranous nephropathy; one with azathioprine; and one with tacrolimus. Three of these patients received a booster vaccine and 3 did not.

Response to BNT162b2 Vaccine Booster in Hemodialysis Patients
All 3 MHD patients who received a booster dose developed a positive IgG S1 titer after the booster dose. IgG $\mathrm{S} 1$ titers increased from $43.5 \pm 45.8 \mathrm{AU} / \mathrm{mL}$ to $6,318.2 \pm$ $6,627.2 \mathrm{AU} / \mathrm{mL}$ in these patients $(p=0.24)$, whereas all patients in the control group remained with low IgG S1 titers $(p=0.42)$. Antibody response to the booster in patients treated with chronic immunosuppression therapy was not inferior to those who were not (mean elevation of $6,274.7 \pm 6,582.2 \mathrm{AU} / \mathrm{mL}$ vs. $16,328.6 \pm 15,318.1 \mathrm{AU} /$ $\mathrm{mL}, p=0.265)$.

\section{Negative IgG S1 Titer against SARS-CoV-2 Spike \\ Protein}

Six months after 2 doses of the BNT162b2 vaccine, 16 out of 102 MHD patients (18.1\%) had IgG S1 lower than $50 \mathrm{AU} / \mathrm{mL}$. After the third dose, all MHD patients in the booster group had a positive IgG S1 titer ( $>50 \mathrm{AU} / \mathrm{mL})$.

In the second measurement, $3(13.6 \%)$ patients in the control group had undetectable IgG titers, while no patient in the booster group had undetectable IgG titers

$(p=0.002)$, odds ratio for an undetected titer was 4.5 (95\% CI 3-6.67) in the control group. The remaining 13 patients with low IgG S1 at the first measurement received a booster that elevated their IgG S1 levels to a mean titer of 4,463.1 \pm 7,036.2 AU/mL (median 2,591.6 range $531.2-27,448 \mathrm{AU} / \mathrm{mL}$ ). None of the patients in the booster group was infected with COVID-19.

Table 3 presents the results of linear regressions that predict the difference between the two IgG S1 measurements. We regressed this difference according to demographics, comorbidities, and baseline laboratory data that were found statistically different between the groups in univariate analyses. After adjusting for demographics, 
Table 3. Standardized regression coefficients predicting delta of IgG S1 titers

\begin{tabular}{llll}
\hline Variable & Total sample & Booster group & Control group \\
\hline Age (years) & 0.08 & 0.08 & 0.18 \\
Sex (male) & -0.02 & -0.03 & -0.33 \\
Hypertension & -0.15 & -0.18 & -0.12 \\
Ischemic heart disease & -0.11 & -0.12 & 0.08 \\
Heart failure & -0.04 & -0.04 & 0.21 \\
Peripheral vascular disease & 0.06 & 0.08 & -0.58 \\
Serum creatinine (mg/dL) & -0.07 & -0.08 & 0.18 \\
Booster group (vs. control) & $0.48^{*}$ & - & - \\
\hline
\end{tabular}

$* p<0.01$. comorbidities, and baseline laboratory data among the total sample, the booster group demonstrated significant increases in IgG S1 levels between measurements $(\beta=$ $0.48, p=0.001$ ).

When examining this model for each group separately, no significant predictors were found for the difference in IgG S1. However, several trends were found. Among the booster group, hypertension was found to predict a smaller improvement $(\beta=-0.18, p=0.20)$. In the control group, hypertension $(\beta=-0.12, p=0.61)$ and $\operatorname{PVD}(\beta=$ $-0.58, p=0.12)$ were found to predict smaller increases.

\section{Discussion}

This study describes the humoral response of MHD patients following a booster dose of the BNT162b2 (Pfizer-BioNTech) vaccine. We compared IgG S1 levels 5 months after the second dose of the vaccine to the levels 17 days after a third (booster dose). Additionally, we compared our findings to a group of MHD patients who did not receive the booster dose, for various reasons.

After performing anti-N Ab (SARS-CoV-2) testing on all patients in the cohort, we were left with 88 patients who were not previously infected with COVID-19. The adverse event profile was similar to that of previous doses and no significant adverse effect was reported.

Our pivotal finding was that following the booster dose, IgG S1 levels increased among 98.5\% of MHD patients, to levels presumably higher than the initial response. The IgG S1 levels were significantly higher (mean 16,336.8 AU/mL) when compared to levels previously published in MHD patients using the same kit (Abbott) we did. While IgG S1 levels in the booster group substantially increased, the IgG S1 levels in the control group continued to decline. Given the current evidence regarding waning immunity and breakthrough infections, this finding supports the recent policy of offering a booster shot to patients vaccinated 5-6 months ago [3-5].

A study by Grupper et al. [7] and other studies [8] showed that a high percentage of MHD patients respond to the BNT162b2 vaccine $96 \%$ and $93 \%$, respectively. However, their antibody levels were significantly lower compared to healthy controls, with a mean level of 3,946 $\mathrm{AU} / \mathrm{mL}$ compared to a mean of $10,144 \mathrm{AU} / \mathrm{mL}$ in healthy controls [7] or a mean of 1,190.8 AU/mL [8] following vaccination with the $B N T 162 \mathrm{~b} 2$ vaccine.

Low albumin levels were associated with a diminished humoral response. Albumin is an important marker of well-being and adequate nutrition in dialysis patients and there are ample reports regarding the influence of malnutrition on the immune system and lymphocyte function $[10,11]$. CRP as a marker of inflammation was also associated with a reduced humoral response, as described previously $[12,13]$.

As described in the Methods section, 6 patients were undergoing chronic immunosuppression therapy. Three received a booster dose and, subsequently, developed a positive IgG S1 titer. Although this is a small number with which to establish general conclusions, it adds to the growing body of literature that demonstrates low levels of seroconversion in immunocompromised patients [14, 15]. Some preliminary articles reported that immunocompromised patients benefitted from a third dose of the Pfizer BNT162b2 vaccine; in agreement with our findings [16].

Linear regression analysis did not indicate a difference in demographics and comorbidities and the chance of developing a significant humoral response across both groups. Recent breakthrough infections have prompted the question whether vaccine efficacy is decreasing due to waning immunity, to vaccine evasion by new SARSCoV-2 variants, i.e., Delta $(\gamma)$ (B.1.617.2), Omicron (B.1.1.529), or both [3]. 
Growing evidence suggests that elapsed time has a major role in breakthrough infections due to waning immunity and declining antibody levels [4]. Bergwerk et al. [5] described breakthrough infections in a cohort of healthcare workers and concluded that SARS-CoV-2 breakthrough infections were correlated with neutralizing antibody titers in the peri-infection period.

Waning immunity could be correlated with the interval between the first and second doses, as suggested by an article (in press) that examined antibody levels in the UK. Although dosage intervals were as long as 8-12 weeks, the group demonstrated a robust response with wider spacing of the doses [17].

The vaccine type might influence the antibody level. Several studies reported better efficacy and antibody levels following the mRNA-1273 (Moderna) among MHD patients [18-21]. Real world data from Israel suggest the booster is effective in restoring vaccine efficacy and reducing infection, as well as hospitalization and severe infection [22-24].

Many vaccines have a schedule of more than 2 doses (i.e., hepatitis B). One might argue that a third dose of the mRNA vaccines should not be considered a booster but should have been part of the original vaccine series. Antibody levels after inactivated vaccines increase after a dose given 4-6 months after the first doses $[25,26]$.

\section{Limitations}

This study had several limitations. We did not examine IgG S1 levels following the second dose; however, there is ample work on the response rate of MHD patients following vaccination and our cohort is presumably no different in this regard.

While we were not able to assess neutralizing antibodies, there seems to be a good correlation between the IgG S1 we examined and neutralizing antibodies $[27,28]$. In addition, we did not examine the cellular arm of the immune system. Despite the relatively small number of patients, we used several statistical analyses to strengthen the validity of our findings as described above, and we believe the findings reported here are applicable to larger cohorts.

\section{Conclusion}

A booster dose of the Pfizer BNT162b2 vaccine effectively increased IgG S1 levels in MHD patients above those found following the second dose. It is safe, with minimal side effects. Antibody levels seem to steadily de- crease by 6 months after a second dose. Precise timing and dosing should be determined by further observational studies, including clinical outcome studies following the booster dose.

\section{Acknowledgment}

We thank Faye Schreiber, MS (Meir Medical Center), for editing the manuscript.

\section{Statement of Ethics}

The study was approved by the local Institutional Ethics Committee in keeping with the principles of the Declaration of Helsinki (MMC 0029-21). Informed consent was obtained from all participants prior to study entry. The study was performed in accordance with the Declaration of Helsinki and Good Clinical Practice guidelines.

\section{Funding Sources}

None to declare.

\section{Author Contributions}

M.S., N.N., A.G., S.B., P.S., and K.C.H.: research area and study design; N.N., D.E., M.L., I.R., O.W., T.H., and K.C.H.: data acquisition; P.S., O.W., S.B., Y.S., and $\mathrm{KCH}$ : data analysis and interpretation; Y.S., O.W., A.G., and K.C.H.: statistical analysis; M.S., S.B., and K.C.H.: supervision or mentorship.

\section{Conflict of Interest Statement}

The authors have no conflicts of interest to declare.

\section{Data Availability Statement}

All data analyzed during this study are included in this article. Further inquiries can be directed to the corresponding author. 


\section{References}

1 Taji L, Thomas D, Oliver MJ, Ip J, Tang Y, Yeung A, et al. COVID-19 in patients undergoing long-term dialysis in Ontario. CMAJ. $2021 \mathrm{Feb} ; 193(8)$ :E278-84.

2 Weekly epidemiological update on COVID-19 - 26 October 2021 [Internet]. [cited 2021 Oct 31]. Available from: https://www. who.int/publications/m/item/weekly-epidemiological-update-on-covid-19---26-october-2021.

3 De-Leon $\mathrm{H}$, Aran D. What pushed Israel out of herd immunity? Modeling COVID-19 spread of delta and waning immunity. medRxiv. 2021 Sep:21263451.

4 Goldberg Y, Mandel M, Bar-On YM, Bodenheimer O, Freedman L, Haas EJ, et al. Waning immunity of the BNT162b2 vaccine: a nationwide study from Israel. medRxiv. 2021 Aug: 21262423.

5 Bergwerk M, Gonen T, Lustig Y, Amit S, Lipsitch $\mathrm{M}$, Cohen $\mathrm{C}$, et al. Covid-19 breakthrough infections in vaccinated health care workers. N Engl J Med. 2021 Oct 14;385(16): 1474-84.

6 FDA authorizes booster dose of pfizer-BioNTech COVID-19 vaccine for certain populations | FDA [Internet]. [cited 2021 Sep 26]. Available from: https://www.fda.gov/newsevents/press-announcements/fda-authorizes-booster-dose-pfizer-biontech-covid19-vaccine-certain-populations.

7 Grupper A, Sharon N, Finn T, Cohen R, Israel $\mathrm{M}$, Agbaria A, et al. Humoral response to the pfizer BNT162b2 vaccine in patients undergoing maintenance hemodialysis. Clin J Am Soc Nephrol. 2021 Jul;16(7):1037-42.

8 Agur T, Ben-Dor N, Goldman S, Lichtenberg S, Herman-Edelstein M, Yahav D, et al. Antibody response to mRNA SARS-CoV-2 vaccine among dialysis patients-a prospective cohort study. Nephrol Dial Transplant. 2021;36: 1347-9.

9 SARS-CoV-2 immunoassay |Abbott core laboratory [Internet]. [cited 2021 Apr 1]. Available from: https://www.corelaboratory.abbott/int/en/offerings/segments/infectiousdisease/sars-cov-2.

10 Prendergast AJ. Malnutrition and vaccination in developing countries. Philos Trans R Soc Lond B Biol Sci. 2015 Jun; 370(1671): 20140141.
11 Rytter MJ, Kolte L, Briend A, Friis H, Christensen VB. The immune system in children with malnutrition: a systematic review. PLoS One. 2014 Aug;9(8):e105017.

12 Pereira B, Xu XN, Akbar AN. Targeting inflammation and immunosenescence to improve vaccine responses in the elderly. Front Immunol. 2020 Oct;11:583019.

13 Pinti M, Appay V, Campisi J, Frasca D, Fülöp $\mathrm{T}$, Sauce D, et al. Highlights review aging of the immune system: focus on inflammation and vaccination. Eur J Immunol. 2016;46: 2286-301.

14 Rabinowich L, Grupper A, Baruch R, Ben-Yehoyada M, Halperin T, Turner D, et al. Low immunogenicity to SARS-CoV-2 vaccination among liver transplant recipients. J Hepatol. 2021 Aug;75(2):435-8.

15 Grupper A, Rabinowich L, Schwartz D, Schwartz IF, Ben-Yehoyada M, Shashar M, et al. Reduced humoral response to mRNA SARS-CoV-2 BNT162b2 vaccine in kidney transplant recipients without prior exposure to the virus. Am J Transplant. 2021;21:271926.

16 Kamar N, Abravanel F, Marion O, Couat C, Izopet J, Del Bello A. Three doses of an mRNA covid-19 vaccine in solid-organ transplant recipients. N Engl J Med. 2021 Jun;385(7):661-

17 Hall V, Ferreira V, Ierullo M, Ku T, Majchrzak-Kita B, Kulasingam V, et al. Delayed interval BNT162b2 mRNA COVID-19 vaccination provides robust immunity. 2021.

18 Kaiser RA, Haller MC, Apfalter P, Kerschner $\mathrm{H}$, Cejka D. Comparison of BNT162b2 (Pfizer-BioNtech) and mRNA-1273 (Moderna) SARS-CoV-2 mRNA vaccine immunogenicity in dialysis patients. Kidney Int. 2021 Sep; 100(3):697-8.

19 Van Praet J, Reynders M, De Bacquer D, Viaene L, Schoutteten MK, Caluwé R, et al. Predictors and dynamics of the humoral and cellular immune response to SARS-CoV-2 mRNA vaccines in hemodialysis patients: a multicenter observational study. J Am Soc Nephrol. 2021 Dec;32(12):3208-20.
20 Garcia P, Anand S, Han J, Montez-Rath ME, Sun S, Shang T, et al. COVID-19 vaccine type and humoral immune response in patients receiving dialysis. J Am Soc Nephrol. 2021 Dec; 33(1):33-7.

21 Stumpf J, Siepmann T, Lindner T, Karger C Schwöbel J, Anders L, et al. Humoral and cellular immunity to SARS-CoV-2 vaccination in renal transplant versus dialysis patients: a prospective, multicenter observational study using mRNA-1273 or BNT162b2 mRNA vaccine. Lancet Reg Health Eur. 2021;9:100178.

22 Levine-Tiefenbrun M, Yelin I, Alapi H, Katz R, Herzel E, Kuint J, et al. Viral loads of Deltavariant SARS-CoV2 breakthrough infections following vaccination and booster with the BNT162b2 vaccine. Nat Med. 2021 Dec: 27(12):2108-10.

23 Bar-On YM, Goldberg Y, Mandel M, Bodenheimer O, Freedman L, Kalkstein N, et al. Protection of BNT162b2 vaccine booster against Covid-19 in Israel. N Engl J Med. 2021 Oct 7;385(15):1393-400.

24 Barda N, Dagan N, Cohen C, Hernán MA, Lipsitch M, Kohane IS, et al. Effectiveness of a third dose of the BNT162b2 mRNA COVID-19 vaccine for preventing severe outcomes in Israel: an observational study. Lancet. 2021 Oct;398(10316):2093-100.

25 Osiowy C. From infancy and beyond: ensuring a lifetime of hepatitis B virus (HBV) vaccine-induced immunityensuring a lifetime of hepatitis B virus (HBV) vaccine-induced immunity. Hum Vaccin Immunother. 2018 May;14(8):2093-7.

26 Venters C, Graham W, Cassidy W, Denham S. Recombivax-HB: perspectives past, present and future. Expert Rev Vaccines. 2014 Apr; 3(2): 119

27 Trougakos IP, Terpos E, Zirou C, Sklirou AD, Apostolakou F, Gumeni S, et al. Comparative kinetics of SARS-CoV-2 anti-spike protein RBD IgGs and neutralizing antibodies in convalescent and naïve recipients of the BNT162b2 mRNA vaccine versus COVID-19 patients. BMC Medicine. 2021 Dec;19(1):208.

28 Wajnberg A, Amanat F, Firpo A, Altman DR, Bailey MJ, Mansour M, et al. Robust neutralizing antibodies to SARS-CoV-2 infection persist for months. Science. 2020 Dec; 370(6521):1227-30. 